\title{
Arbitrary scattering of an electromagnetic zero-order Bessel beam by a dielectric sphere
}

\author{
F. G. Mitri \\ Los Alamos National Laboratory, MPA-11, Sensors \& Electrochemical Devices, Acoustics \& Sensors Technology Team, \\ MS D429, Los Alamos, New Mexico 87545, USA (mitri@lanl.gov)
}

Received November 29, 2010; revised February 1, 2011; accepted February 2, 2011; posted February 4, 2011 (Doc. ID 138883); published March 1, 2011

\begin{abstract}
Arbitrary electromagnetic (EM) scattering of a zero-order Bessel beam by a homogeneous water sphere in air is investigated. The radial components of the electric and magnetic scattering fields are expressed using a partial wave series involving the beam-shape coefficients, scattering coefficients of the sphere, and half-conical angle of the wavenumber components of the beam. The 3D scattering directivity plots in the far-field region are evaluated using a numerical integration procedure. It is shown here that shifting the sphere off the axis of wave propagation breaks the symmetry in the directivity patterns. Moreover, the scattering strongly depends on the half-cone angle of the beam. This investigation could provide a useful test of finite element codes for the evaluation of EM scattering and radiation forces, which are important in optical tweezers and related particle manipulation applications. @ 2011 Optical Society of America

OCIS codes: $\quad 260.2110,290.5825,290.5850$.
\end{abstract}

Just as such early works on the axial scattering of an infinite plane EM wave [1-4] and the on- and off-axial scattering of a Gaussian beam [5,6] incident upon a homogeneous sphere were made available in the literature, rigorous analytical solutions for on- and off-axial scattering of an EM zero-order Bessel beam (ZOBB) by a (homogeneous) spherical particle seem to be nonexistent. The fundamental Bessel EM eigenbeam possesses some advantages over conventional Gaussian (or other) beams $[\underline{7}, \underline{8}]$. Thus, it provides an impetus to investigate its EM scattering properties by a homogeneous water sphere in air.

Based on the studies presented in [6,9], the radial components of the (steady-state) scattered electric and magnetic fields can be expressed, respectively, as

$$
\begin{aligned}
E_{r}^{s c}(r, \theta, \phi)= & \frac{a^{2}}{r^{2}} \sum_{n=1}^{\infty} \sum_{m=-n}^{n} n(n+1) a_{n m}(k a) \\
& \times \xi_{n}^{(1)}(k r) Y_{n}^{m}(\theta, \phi), \\
H_{r}^{s c}(r, \theta, \phi)= & \frac{a^{2}}{r^{2}} \sum_{n=1}^{\infty} \sum_{m=-n}^{n} n(n+1) b_{n m}(k a) \\
& \times \xi_{n}^{(1)}(k r) Y_{n}^{m}(\theta, \phi),
\end{aligned}
$$

where $k=k_{0} \varepsilon^{-1 / 2}, k_{0}=\omega / c$, where $\omega$ and $c$ are the angular frequency and speed of the EM wave in vacuum, $\varepsilon \neq 0$ is the dielectric constant of the medium, $a$ is the radius of the sphere, $r$ is the distance to a point in space, $\xi_{n}^{(1)}($.$) is$ the spherical Riccati-Hankel function of the first kind, $Y_{n}^{m}(\theta, \phi)$ are the spherical harmonics, and $a_{n m}$ and $b_{n m}$ are the coupled scattering/beam-shape coefficients given by $[\underline{6}, \underline{9}]$ :

$$
\begin{aligned}
& a_{n m}=\left[\frac{\psi_{n}^{\prime}(\bar{n} k a) \psi_{n}(k a)-\bar{n} \psi_{n}(\bar{n} k a) \psi_{n}^{\prime}(k a)}{\bar{n} \psi_{n}(\bar{n} k a) \xi_{n}^{(1) \prime}(k a)-\psi_{n}^{\prime}(\bar{n} k a) \xi_{n}^{(1)}(k a)}\right] A_{n m}, \\
& b_{n m}=\left[\frac{\bar{n} \psi_{n}^{\prime}(\bar{n} k a) \psi_{n}(k a)-\psi_{n}(\bar{n} k a) \psi_{n}^{\prime}(k a)}{\psi_{n}(\bar{n} k a) \xi_{n}^{(1) \prime}(k a)-\bar{n} \psi_{n}^{\prime}(\bar{n} k a) \xi_{n}^{(1)}(k a)}\right] B_{n m},
\end{aligned}
$$

where $\psi_{n}($.$) , is the spherical Riccati-Bessel function of$ the first kind, the prime denotes a derivative with respect to the argument, the parameter $\bar{n}$ is the relative refractive index of the medium of wave propagation (which is generally complex), and the beam-shape coefficients $A_{n m}$ and $B_{n m}$ are given, respectively, by

$$
\begin{aligned}
A_{n m}(k a)= & \frac{1}{n(n+1) \psi_{n}(k a)} \\
& \times \int_{0}^{2 \pi} \int_{0}^{\pi} \sin \theta E_{r}^{\mathrm{inc}}(a, \theta, \phi) Y_{n}^{m *}(\theta, \phi) \mathrm{d} \theta \mathrm{d} \phi,
\end{aligned}
$$

$$
\begin{aligned}
B_{n m}(k a)= & \frac{1}{n(n+1) \psi_{n}(k a)} \\
& \times \int_{0}^{2 \pi} \int_{0}^{\pi} \sin \theta H_{r}^{\mathrm{inc}}(a, \theta, \phi) Y_{n}^{m *}(\theta, \phi) \mathrm{d} \theta \mathrm{d} \phi,(6)
\end{aligned}
$$

where the superscript $*$ denotes a complex conjugate, and $E_{r}^{\mathrm{inc}}(r=a, \theta, \phi)$ and $H_{r}^{\mathrm{inc}}(r=a, \theta, \phi)$ are the radial (scalar) components of the incident electric and magnetic fields evaluated at the radius of the sphere.

As noted in Eqs. (5) and (6), the incident electric and magnetic fields need to be determined to properly solve for the scattering problem. One may use the scalar wave theory [10], which provides fairly good results only if the size of the central spot of the beam is much larger than the wavelength [i.e., $k_{R}(=k \sin \beta) \ll k$, where $\beta$ is the half-cone angle of the beam]. However, it has been shown [11] that the vector nature of electromagnetic (EM) wave propagation introduces significant corrections and should be used instead for a complete analysis of circularly symmetric beams, such as the ZOBB. Therefore, the radial components of the incident electric and magnetic fields are expressed in terms of the Cartesian coordinates, respectively, as $[\underline{12}, \underline{13}]$ 


$$
\begin{aligned}
\left\{\begin{array}{l}
E_{r}^{\text {inc }}(a, \theta, \phi) \\
H_{r}^{\text {inc }}(a, \theta, \phi)
\end{array}\right\}= & \left\{\begin{array}{l}
E_{x}^{\text {inc }} \\
H_{x}^{\text {inc }}
\end{array}\right\}_{r=a} \sin \theta \cos \phi \\
& +\left\{\begin{array}{l}
E_{y}^{\text {inc }} \\
H_{y}^{\text {inc }}
\end{array}\right\}_{r=a} \sin \theta \sin \phi \\
& +\left\{\begin{array}{l}
E_{z}^{\text {inc }} \\
H_{z}^{\text {inc }}
\end{array}\right\}_{r=a} \cos \theta,
\end{aligned}
$$

where [11]

$$
\begin{aligned}
& E_{x}^{\mathrm{inc}}=\frac{1}{2} E_{0}\left[\left(1+\frac{k_{z}}{k}-\frac{k_{R}^{2} x^{2}}{k^{2} R^{2}}\right) J_{0}\left(k_{R} R\right)\right. \\
& \left.-\frac{k_{R}\left(y^{2}-x^{2}\right)}{k^{2} R^{3}} J_{1}\left(k_{R} R\right)\right] \exp \left(i k_{z} z\right), \\
& E_{y}^{\mathrm{inc}}=\frac{1}{2} E_{0} x y\left[\frac{2 k_{R}}{k^{2} R^{3}} J_{1}\left(k_{R} R\right)\right. \\
& \left.-\left(\frac{k_{R}^{2}}{k^{2} R^{2}}\right) J_{0}\left(k_{R} R\right)\right] \exp \left(i k_{z} z\right), \\
& E_{z}^{\mathrm{inc}}=\frac{1}{2 i} E_{0} \frac{x}{k R}\left(1+\frac{k_{z}}{k}\right) k_{R} J_{1}\left(k_{R} R\right) \exp \left(i k_{z} z\right) . \\
& H_{x}^{\mathrm{inc}}=\sqrt{\varepsilon} E_{y}^{\mathrm{inc}}, \\
& H_{y}^{\mathrm{inc}}=\sqrt{\varepsilon} \frac{1}{2} E_{0}\left[\left(1+\frac{k_{z}}{k}-\frac{k_{R}^{2} y^{2}}{k^{2} R^{2}}\right) J_{0}\left(k_{R} R\right)\right. \\
& \left.-\frac{k_{R}\left(x^{2}-y^{2}\right)}{k^{2} R^{3}} J_{1}\left(k_{R} R\right)\right] \exp \left(i k_{z} z\right), \\
& H_{z}^{\mathrm{inc}}=\sqrt{\varepsilon} \frac{1}{2 i} E_{0} \frac{y}{k R}\left(1+\frac{k_{z}}{k}\right) k_{R^{e}} J_{1}\left(k_{R} R\right) \exp \left(i k_{z} z\right) .
\end{aligned}
$$

The parameter $E_{0}=i k A_{0}, k_{z}=k \cos \beta, R=\sqrt{x^{2}+y^{2}}$ is the radial distance to a point in the transverse plane $(x, y)$, and $J_{0,1}(\cdot)$ is the cylindrical Bessel function of the first kind of the zeroth, and first orders, respectively.
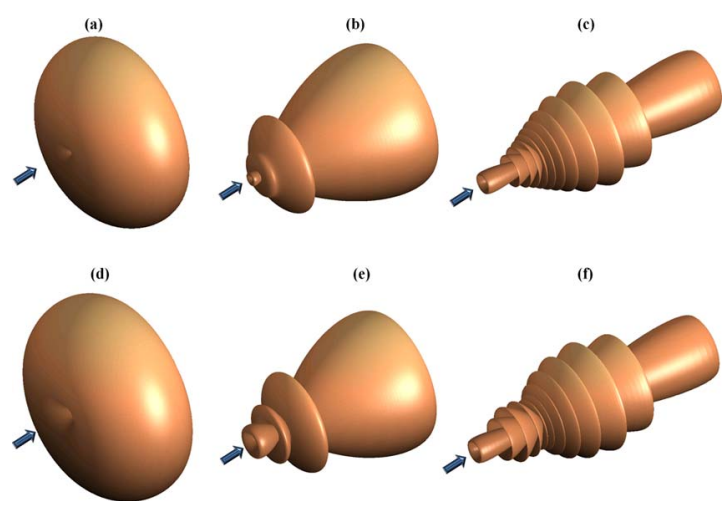

Fig. 1. (Color online) Magnitude of the far-field scattering form functions of a plane EM wave (i.e., $\beta=0^{\circ}$ ) incident upon a water sphere for the scattered electric [(a)-(c)] and magnetic [(d)-(f)] far fields. The plots in (a), (d) correspond to $k a=1.5$; (b), (e) to $k a=5$; and (c), (f) to $k a=12$, respectively.

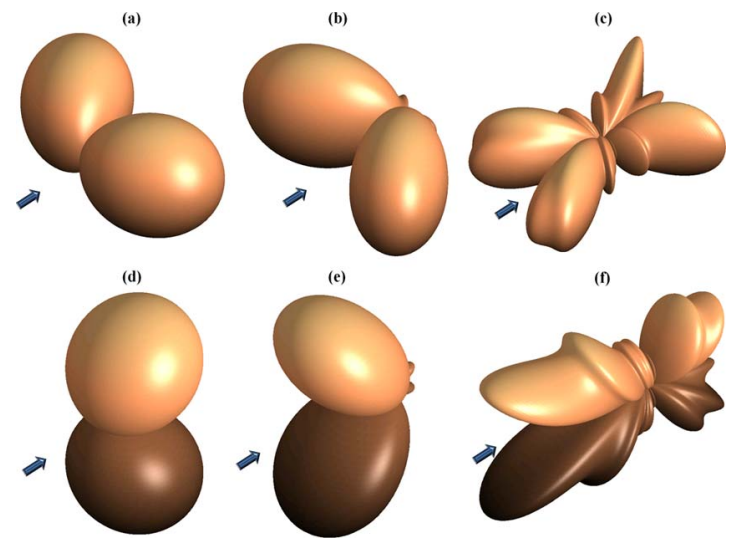

Fig. 2. (Color online) Magnitude of the far-field scattering form functions for a zero-order Bessel EM beam with $\beta=55^{\circ}$, for the scattered electric [(a)-(c)] and magnetic [(d)-(f)] far fields. The plots in (a), (d) correspond to $k a=1.5,(\mathrm{~b}),(\mathrm{e})$ to $k a=5$; and (c), (f) to $k a=12$, respectively.

It is common to investigate the scattered electric and magnetic fields in the far-field region, though Eqs. (1) and (2) can be used to compute the scattered fields at any distance $r$ from the sphere. In the far field $(k r \rightarrow \infty)$, the expression for the scattered field is further simplified because

$$
\left\{\begin{aligned}
\psi_{n}(q) & \rightarrow \sin (q-n \pi / 2), \\
\xi_{n}^{(1)}(q) & \rightarrow i^{-(n+1)} \exp (i q) .
\end{aligned}\right.
$$

In that limit, a scattering form function for the electric field may be defined as

$$
f_{\infty}^{E}(k a, \theta, \phi)=\sum_{n=1}^{\infty} \sum_{m=-n}^{n} n(n+1) i^{-(n+1)} a_{n m} Y_{n}^{m}(\theta, \phi),
$$

and similarly for the magnetic field as

$$
f_{\infty}^{H}(k a, \theta, \phi)=\sum_{n=1}^{\infty} \sum_{m=-n}^{n} n(n+1) i^{-(n+1)} b_{n m} Y_{n}^{m}(\theta, \phi) .
$$

Upon the substitution of Eqs. (3) and (4) into Eqs. (15) and (16) using Eqs. (5)-(13), the magnitude (or phase) of the scattering electric and magnetic form functions can be evaluated by plotting the on- and off-axial 3D
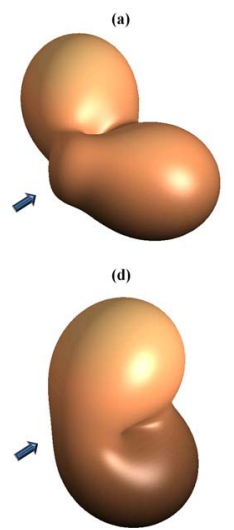

Fig. 3. (Color online) The same as in Fig. 3; however, the sphere is shifted off the axis of the incident beam in both the $x$ and $y$ directions such that the offset (in arbitrary units) is $(x, y)$ - offset $=(0.75 ; 0.75)$. 
directivity patterns. Calculation of the beam-shape coefficients requires determining the surface integral in Eqs. (5) and (6). The integrals are evaluated by quadrature based on a Riemann sum in the MATLAB software package. It is important to emphasize that dense grids (sampled here at $2^{25}$ uniformly distributed points) are required in both the $\theta$ and $\phi$ directions to obtain proper convergence of the numerical integrations. In the simulations, the value of the parameters are $E_{0}=\varepsilon=1$, and the (complex) refractive index of refraction of the medium is $\bar{n}=1.33+5 \times 10^{-6} i[14]$.

To validate and verify the accuracy of the computations, a test for a linearly polarized (in the $x$ direction) plane EM wave has been performed to compute the beam-shape coefficients for the electric field using Eq. (5) and we compared the results with the exact known solution. The same can be done for the magnetic field using Eq. (6); however, it has not been reported here for brevity. After some arithmetic manipulation based on the expression for the radial component of the incident electric field of a plane EM wave given by (7.75) in [15] and its comparison with (A1) in [9], the beamshape coefficients can be identified and determined as

$$
\left.A_{n m}(k a)\right|_{\text {plane wave }}=\frac{i^{(n+1)}}{(k a)^{2}} \sqrt{\frac{\pi(2 n+1)}{n(n+1)}} \delta_{m 1},
$$

where the Kronecker delta $\delta_{m 1}=\left\{\begin{array}{c}1, m=1 \\ 0, m \neq 1\end{array}\right.$. The computed beam-shape coefficients using (5) have been compared to those obtained from the exact form as given by Eq. (17). The relative numerical error was about $\sim 10^{-7}$. Also, computations for the magnitude of Eqs. (15) and (16) are performed for the axial scattering of a plane $\mathrm{EM}$ wave by a homogeneous sphere by setting $\beta=0^{\circ}$ with $E_{0}=\varepsilon=1$ in Eqs. (8)-(13).

The results are shown in Figs. 1(a)-1(f) for the radial components of the scattered electric and magnetic fields, respectively. As observed, the magnitude of the scattering by the sphere along the axis vanishes. This is somewhat expected because both radial components of the electric and magnetic fields depend on the associated Legendre function of the first order $P_{n}^{1}(\cos \theta)$ \{see (7.75) and (7.77) in [15]\} that vanishes for $\theta=0$ and $\theta= \pm \pi$. The arrows in Fig. 1 (and others) on the left-hand side of each panel indicate the direction of the incident waves $(\theta=\pi)$.

The case of a ZOBB EM beam incident upon a water sphere is represented in Fig. 2, which shows a comparison between the 3D scattering directivity patterns for the magnitude of the electric and magnetic far-field form functions as given in Eqs. (15) and (16). The plots correspond to the scattering far fields by a sphere centered on the axis of the ZOBB. One notices the significant differences as compared to the plane wave result (i.e., Fig. 1). Moreover, the 3D scattering directivity patterns show spatial symmetry with respect to the center of the sphere.

This is not the case when the water sphere is shifted off the axis of the EM ZOBB. For this example, the sphere is shifted in both the $x$ and $y$ directions, such that the offset in arbitrary units is $(x, y)$ - offset $=(0.75 ; 0.75)$.
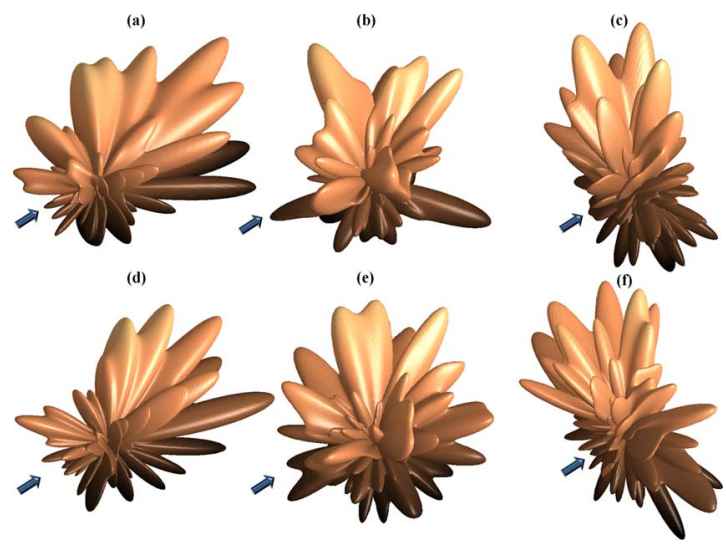

Fig. 4. (Color online) Magnitude of the far-field scattering form functions for the scattered electric [(a)-(c)] and magnetic [(d)-(f)] far fields from a homogeneous water sphere placed off axially $[(x, y)$ - offset $=(0.75 ; 0.75)]$ with respect to the axis of an incident zero-order Bessel EM beam at $k a=12$ for various values of the half-cone angle $\beta$. In (a), (d) $\beta=15^{\circ}$, in (b), (e) $\beta=40^{\circ}$, and in (c), (f) $\beta=75^{\circ}$, respectively.

It is obvious that the 3D scattering directivity patterns for the off-axial scattering of the electric and magnetic far fields displayed in Fig. 3 show significant differences from the axial case displayed in Fig. 2.

Further calculations for the off-axial far-field electric and magnetic scattering are performed to investigate the effect of varying the half-cone angle $\beta$ on the 3D scattering directivity patterns. The results are displayed in Fig. 4, in which the off-axial scattering directivity patterns in the far field are sensitive to the variations of the half-cone angle $\beta$. In contrast to the case of the plane waves, both the axial and off-axial scattering from an EM ZOBB are strongly affected by the value of the half-cone angle $\beta$.

\section{References}

1. G. Mie, Ann. Phys. 330, 377 (1908).

2. H. C. van de Hulst, Light Scattering by Small Particles (Wiley, 1957).

3. P. W. Dusel, M. Kerker, and D. D. Cooke, J. Opt. Soc. Am. 69, 55 (1979).

4. R. G. Newton, Scattering Theory of Waves and Particles, 2nd ed. (Springer-Verlag, 1982).

5. J. A. Lock, Appl. Opt. 34, 559 (1995).

6. J. P. Barton, D. R. Alexander, and S. A. Schaub, J. Appl. Phys. 64, 1632 (1988).

7. J. Durnin, J. Miceli, and J. H. Eberly, Phys. Rev. Lett. 58, 1499 (1987).

8. Z. Bouchal, J. Wagner, and M. Chlup, Opt. Commun. 151, 207 (1998).

9. J. P. Barton, D. R. Alexander, and S. A. Schaub, J. Appl. Phys. 66, 4594 (1989).

10. Z. Bouchal and M. Olivík, J. Mod. Opt. 42, 1555 (1995).

11. S. R. Mishra, Opt. Commun. 85, 159 (1991).

12. S. A. Schaub, J. P. Barton, and D. R. Alexander, Appl. Phys. Lett. 55, 2709 (1989).

13. S. A. Schaub, J. P. Barton, and D. R. Alexander, Appl. Phys. Lett. 59, 1798 (1991).

14. G. M. Hale and M. R. Querry, Appl. Opt. 12, 555 (1973).

15. T. Rother, in Electromagnetic Wave Scattering on Nonspherical Particles (Springer, 2009), pp. 197-233. 